\title{
Isospin effect on nuclear stopping in intermediate energy Heavy Ion Collisions *
}

\author{
Qingfeng $\mathrm{Li}^{1)}$, Zhuxia $\mathrm{Li}^{1,2,3)}$ \\ 1) China Institute of Atomic Energy, P. O. Box 275 (18), Beijing 102413, P. R. China \\ 2) Institute of Theoretical Physics, Academia Sinica, P. O. Box 2735, Beijing 100080, P. R. \\ China \\ 3) Center of Theoretical Nuclear Physics, National Laboratory of Lanzhou Heavy Ion \\ Accelerator, Lanzhou 730000, P. R. China
}

\begin{abstract}
By using the Isospin Dependent Quantum Molecular Dynamics Model (IQMD), we study the dependence of nuclear stopping $Q_{Z Z} / A$ and $R$ in intermediate energy heavy ion collisions on system size, initial $N / Z$, isospin symmetry potential and the medium correction of two-body cross sections. We find the effect of initial $N / Z$ ratio, isospin symmetry potential on stopping is weak. The excitation function of $Q_{Z Z} / A$ and $R$ depends on the form of medium correction of two-body cross sections, the equation of state of nuclear matter (EOS). Our results show the behavior of the excitation function of $Q_{Z Z} / A$ and $R$ can provide clearer information of the isospin dependence of the medium correction of two-body cross sections.
\end{abstract}

PACS numbers: 25.70.-z, 24.10.-i

It is important to investigate whether an equilibrium is reached or not for a colliding system in order to obtain the information of EOS and the reaction mechanism correctly. This problem has been studied extensively both theoretically and experimentally [1]-[6]. Following the establishment of radioactive beam facilities in many laboratory, it becomes possible to study neutron (or proton) rich nuclear collisions at intermediate energies. Therefore it is necessary to study the effect of isospin asymmetry on the equilibrium process in a colliding system. In [7] the sensitivity of nuclear stopping to the isospin dependence of cross section was studied but the isospin dependence of the medium correction of two-body cross sections and the isospin asymmetry of the initial system was not

\footnotetext{
*Supported by National Natural Science Foundation of China under No. 19975073, National Science Foundation of Nuclear Industry under No. Y7100A0101 and Major State Basic Research Development Program in China under contract No. G20000774
} 
tested. As we expect the degree of equilibrium is influenced by the mean field, medium correction of two-body cross section as well as the size of colliding system. To considering the influence of the isospin dependence of the medium correction of two-body cross section, in this letter we use 4 different forms of in-medium two-body cross sections, they are: 1) $\sigma_{0}$, the free nucleon-nucleon cross section which is isospin dependent [8], 2) $\sigma_{0}^{*}[9]$, which reads as

$$
\sigma^{*}=\left[1+\alpha\left(\rho / \rho_{0}\right)\right] \sigma^{\text {free }},
$$

here $\alpha=-0.2$. 3) $\sigma_{1}$ according to Ref. [10]. 4) $\sigma_{2}$, in which the medium correction is obtained based on the results of [11]. In [10] G. Q. Li and R. Machleidt calculated the in-medium neutron-proton, proton-proton (neutron-neutron) scattering cross section at energies up to $300 \mathrm{AMeV}$ with the Dirac-Brueckner Hartree-Fock approach where the medium correction of $\rho$ meson mass was not taken into account and it was found that the in-medium cross sections decreased as density and increased slightly when density was higher than normal nuclear density and energy was higher than about $125 \mathrm{AMeV}$ (in Lab. system). In [11] the in-medium binary scattering cross section is derived based on QHD-II type Lagrangian within the framework of the microscopic transport theory at energies up to $800 \mathrm{AMeV}$ where the medium correction of $\rho$ meson mass was taken into account [12] and it was found that because of the medium correction of $\rho$ meson mass the medium correction of nucleon-nucleon cross section was also isospin dependent, i.e., $\sigma_{n p}^{*}$ depends on the baryon density weakly while $\sigma_{n n(p p)}^{*}$ depends on the baryon density obviously and a slightly increasing with energy when density was higher than normal density and energy was higher than about $200 \mathrm{AMeV}$ (in Lab. system) was also shown. The influence of the mean field is considered by taking EOS to be 'soft' EOS $(K=200 \mathrm{MeV})$ and 'hard' EOS $(K=380 M e V)$ as well as with and without isospin symmetry potential. In order to study the dependence of the degree of equilibrium on the nuclear size and the initial isospin asymmetry, we calculate two groups of colliding systems 1) ${ }^{58} \mathrm{Ni}+{ }^{58} \mathrm{Ni}$ and its neighbor nuclei with $A=60 Z=24-32$ and 2) ${ }^{120} S n+{ }^{120} S n$ and its neighbor nuclei with $A=120 Z=47-55$.

We study the degree of equilibrium in momentum space by investigating the momentum quadrupole $Q_{Z Z}$ and $R$, the ratio between perpendicular momentum and parallel momentum, which usually are called nuclear stopping. $Q_{Z Z}$ is defined as

$$
Q_{Z Z}=\Sigma_{i}\left[2 * P_{z}(i)^{2}-P_{x}(i)^{2}-P_{y}(i)^{2}\right]
$$

and $R$ is defined as

$$
R=\frac{2}{\pi}\left(\Sigma_{i}\left|P_{\perp}(i)\right|\right) /\left(\Sigma_{i}\left|P_{z}(i)\right|\right) .
$$

Here the summation runs over all nucleons in projectile and target and the transverse momentum $P_{\perp}(i)=\sqrt{P_{x}(i)^{2}+P_{y}(i)^{2}}$.

The Isospin Dependent Quantum Molecular Dynamics (QMD) model [13, 14] is used in the calculations. For details, please see ref. [15]. The used parameters of EOS are shown in Table 1.

First of all, we investigate the effect of symmetry potential and isospin dependence of the medium correction of two-body cross section as well as the isospin asymmetry of 
initial system on $Q_{Z Z}$. Fig. 1 shows average $Q_{Z Z}$ as function of $\mathrm{Z}$ for projectile and target taken to be a) $A=60, Z$ ranging from 24 to 32 and b) $A=120, Z$ ranging from 47 to 55 at beam energy $50 \mathrm{AMeV}, 150 \mathrm{AMeV}$ and $400 \mathrm{AMeV}$ with $C_{S}=0$ and $35 \mathrm{MeV}$ and two-body cross section taking to be $\sigma_{0}$ and $\sigma_{2}$, respectively. As a whole, we can find that the effects of symmetry potential and the initial isospin asymmetry on $Q_{Z Z}$ are weak for both in-medium cross sections taken to be $\sigma_{0}$ (no medium correction) and $\sigma_{2}$ (with isospin dependent medium correction) cases.

$$
\text { Fig. } 1 \text { a), b) }
$$

In Figs. 2 a) and b) we present the excitation function of average $Q_{Z Z}$ with different EOS ('Soft' and 'Hard') and cross sections $\left(\sigma_{0}, \sigma_{0}^{*}, \sigma_{1}\right.$ and $\left.\sigma_{2}\right)$ for a) ${ }^{58} \mathrm{Ni}+{ }^{58} \mathrm{Ni}$ and b) ${ }^{120} \mathrm{Sn}+{ }^{120} \mathrm{Sn}$. It is shown that $Q_{Z Z} / A$ first increases with beam energy then it is saturated at about $150-300 \mathrm{AMeV}$ depending on the in-medium cross section used. Different forms of medium correction of two-body cross sections significantly affect the behavior of excitation function of $Q_{Z Z} / A$. From comparison between Fig. 2 a) and b) we find that for ${ }^{58} \mathrm{Ni}+{ }^{58} \mathrm{Ni}$ case the influence of different EOS on $Q_{Z Z} / A$ is much weaker than that of different form of cross sections while for ${ }^{120} S n+{ }^{120} S n$ case it is comparable with that of different cross sections. So from above study we may conclude that the behavior of the excitation function of $Q_{Z Z} / A$ for medium size system like ${ }^{58} N i+{ }^{58} N i$ can provide clearer information of isospin dependence of the medium correction of cross sections.

$$
\text { Fig. } 2 \text { a), b) }
$$

Figs. $3 \mathrm{a}$ ) and b) show the excitation function of $R$ for a) ${ }^{58} \mathrm{Ni}+{ }^{58} \mathrm{Ni}$ and b) ${ }^{120} \mathrm{Sn}+{ }^{120}$ $S n$ with different EOS ('Soft' and 'Hard'), cross section $\left(\sigma_{0}, \sigma_{0}^{*}\right.$, and $\left.\sigma_{2}\right)$. For both ${ }^{58} \mathrm{Ni}+{ }^{58} \mathrm{Ni}$ and ${ }^{120} \mathrm{Sn}+{ }^{120} \mathrm{Sn}$ cases, $R$ decreases when beam energy is less than $\sim$ $100 \mathrm{AMeV}$ and there is a change of the decreasing slope at $50 \mathrm{AMeV}$. As beam energy further increases $R$ increases. For ${ }^{58} N i+{ }^{58} N i$ case, the slope of excitation function of $R$ is dominated by the form of in-medium cross sections and its dependence on the mean field is very weak. While for ${ }^{120} S n+{ }^{120} S n$ case, the slope of excitation function of $R$ depends on both form of medium correction of two-body cross section and the EOS of nuclear matter. This behavior of $R$ depending on system size is the same as $Q_{Z Z} / A$ case shown in Figs. 2 a) and b). It implies that the measurement of the excitation function of the stopping in medium size system like ${ }^{58} \mathrm{Ni}+{ }^{58} \mathrm{Ni}$ can provide clearer information of medium correction of two-body cross section.

$$
\text { Fig. } 3 \text { a), b) }
$$

Fig. 4 shows the excitation function of $R$ for ${ }^{120} S n+{ }^{120} S n$ at different impact parameters. The behavior of $R$ as function of energy changes quickly for different impact parameters. The slopes of $R$ as function of beam energy change sign from negative to positive at $E \sim 100 \mathrm{AMeV}$ for $b=0 \mathrm{fm}-b=3 \mathrm{fm}$ cases. For low beam energy the slope 
of excitation function depends on impact parameter weakly. It implies that for lower energy case the stopping does not depend on impact parameter strongly but as energy increases the stopping does depend on impact parameter strongly.

\section{Fig. 4}

In summary, in this paper we study the dependence of nuclear stopping $Q_{Z Z} / A$ and $R$ on isospin asymmetry of the initial system, the isospin dependence of the medium correction of two-body scattering cross section and EOS of nuclear matter (isospin dependent part and incompressibility). We find that the dependence of stopping on initial isospin asymmetry and isospin dependent part of nuclear potential is weak and the dependence on the different forms of the medium correction of two-body cross section is strong. By studying the excitation function of $Q_{Z Z} / A$ and $R$ we find the influence of difference of EOS (incompressibility) on stopping is comparable with that of different medium correction of two-body cross sections for ${ }^{120} \mathrm{Sn}+{ }^{120} \mathrm{Sn}$ but is much weak for ${ }^{58} \mathrm{Ni}+{ }^{58} \mathrm{Ni}$ case at energy range studied in this work. Therefore the measurement of the excitation function of nuclear stopping for collisions of medium size nuclei is a better means to extract the information of medium correction of two-body cross section. We also find that the dependence of nuclear stopping on impact parameter is weak at low energy and it becomes stronger at energy higher than $\sim 50-100 \mathrm{AMeV}$.

Table. 1 Parameters used in calculations

\begin{tabular}{|l|c|c|c|c|c|c|c|}
\hline EOS & $\alpha(\mathrm{MeV})$ & $\beta(\mathrm{MeV})$ & $\gamma$ & $\rho_{0}\left(\mathrm{fm}^{-3}\right)$ & $K(\mathrm{MeV})$ & $L(\mathrm{fm})$ & $C_{Y u k}(\mathrm{MeV})$ \\
\hline Soft & -356 & 303 & $7 . / 6$. & 0.168 & 200 & 2.1 & -5.5 \\
\hline Hard & -124 & 70.5 & 2. & 0.168 & 380 & 2.1 & -5.5 \\
\hline
\end{tabular}

\section{References}

[1] J. Cugnon, T. Mizutani and J. Vandermeulen 1981 Nucl. Phys. A 359 345c

[2] J. Randrup 1979 Nucl. Phys. A 314429

[3] M. Cubero, M. Schonhofen, B. L. Frimen and W. Norenberg 1991 Nucl. Phys. A 340 385

[4] A. Lang, B. Blattel, V. Koch, K. Weber, W. Cassing and U. Mosel 1991 Z. Phys. A 340287

[5] Li Zhuxia, Zhuo Yizhong, Gu Yingqi, et al 1993 Nucl. Phys. A 559603 
[6] Li Zhuxia, Zhuo Yizhong, Wu Xizhen, M. Sano 1994 J. Phys. G: Nucl. Part. Phys. 201829

[7] Jian-Ye Liu, Wen-Jun Guo Shun-JIn Wang, et al 2001 Phys. Rev. Lett. 86975

[8] K. Chen, Z. Fraenkel, G. Friedlander, et al 1968 Phys. Rev. 166949

[9] D. Klakow, G. Wilke and W. Bauer 1993 Phys. Rev. C 481982

[10] G. Q. Li and R. Machleidt 1993 Phys. Rev. C 481702 and 1994 Phys. Rev. C 49 566

[11] Q. F. Li, Z. X. Li and G.J. Mao 2000 Phys. Rev. C 62014606

[12] G. J. Lolos, et al 1998 Phys. Rev. Lett. 80241

[13] J. Aichelin 1991 Phys. Rep. 202233 and references therein.

[14] C. Hartnack, et al 1989 Nucl. Phys. A 495303

[15] Q.F. Li and Z.X. Li 2001 accepted by Phys. Rev. C 


\section{CAPTIONS}

Fig. 1 The average $Q_{Z Z}$ as function of $Z$ for projectile and target taken to be a) $A=60$ and $Z$ ranging from 24 to 32 and b) $A=120, Z$ ranging from 47 to 55 at beam energy $50 \mathrm{AMeV}, 150 \mathrm{AMeV}$ and $400 \mathrm{AMeV}$ with $C_{S}=0$ and $35 \mathrm{MeV}$ and two-body cross section taking to be $\sigma_{0}$ and $\sigma_{2}$ (see text), respectively.

Fig. 2 The average $Q_{Z Z}$ as function of beam energy for a) ${ }^{58} N i+{ }^{58} N i$ and b) ${ }^{120} S n+{ }^{120}$ $S n$ at $b=0 \mathrm{fm}$ with different EOS and two-body cross sections $\sigma$ (see text).

Fig. $\quad 3 R$ as function of beam energy at different EOS and two-body cross sections $\sigma$ (see text).

Fig. $\quad 4 R$ as function of beam energy with different input parameters $b$. 

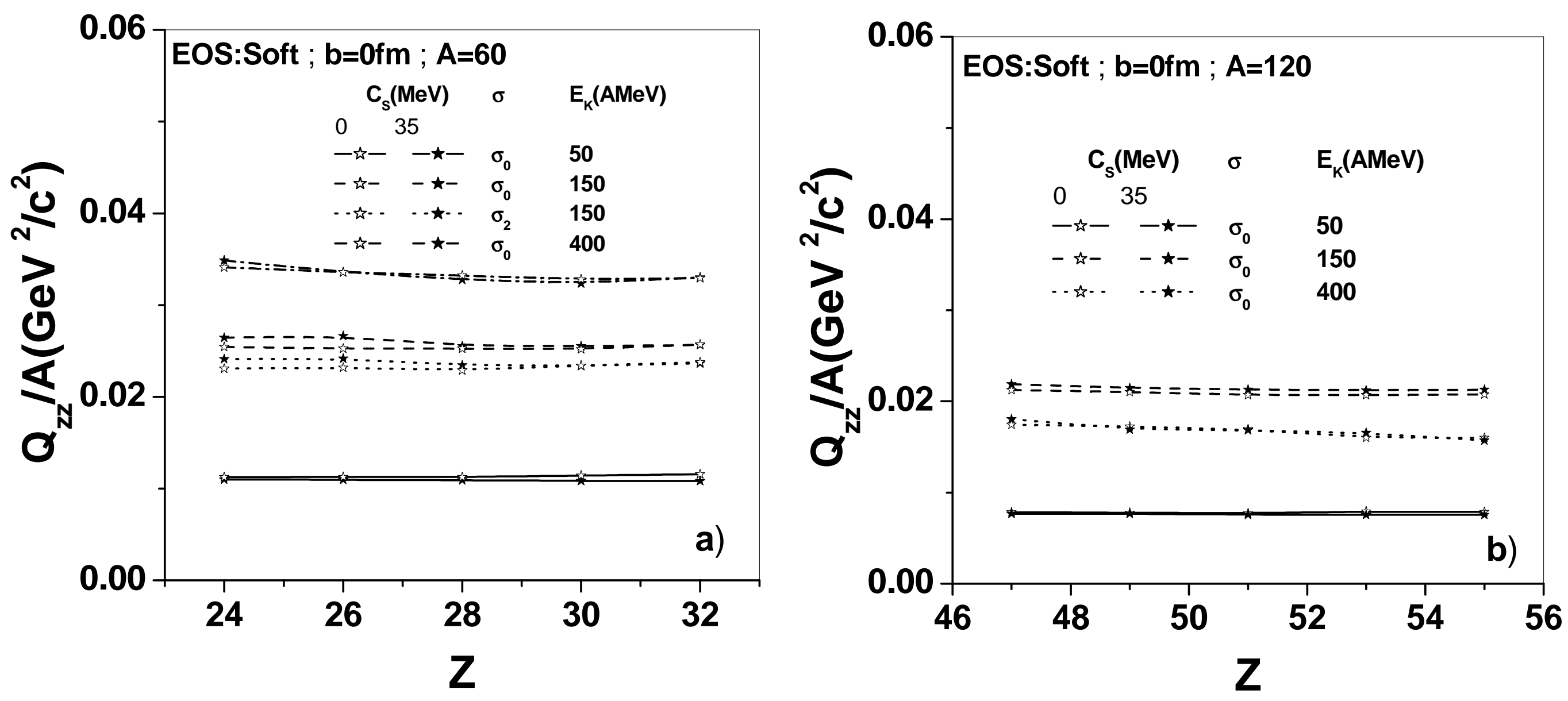

Fig. 1 

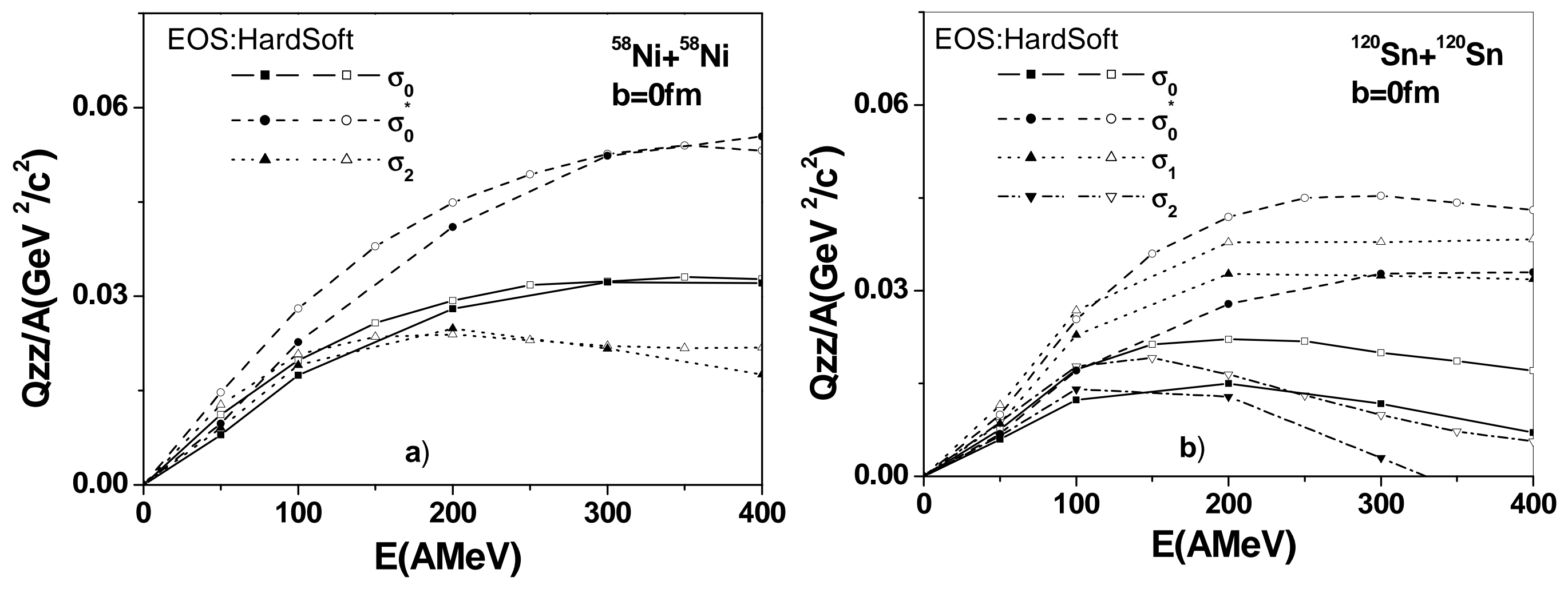

Fig. 2 

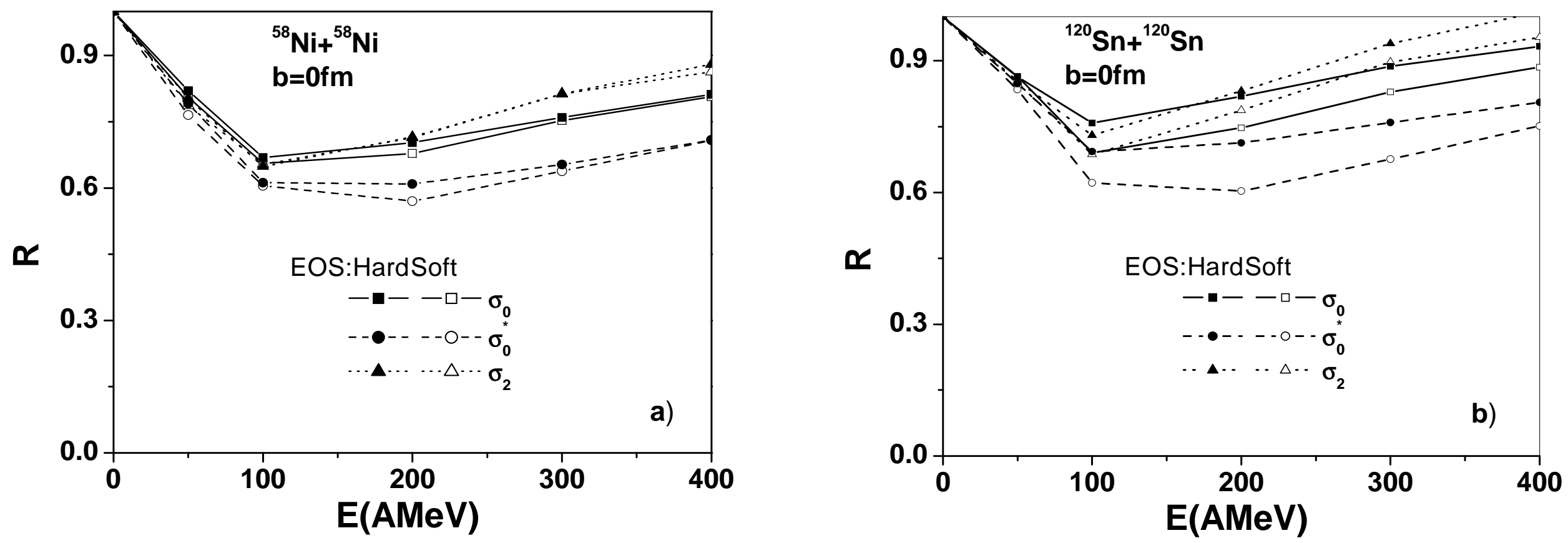

Fig. 3 


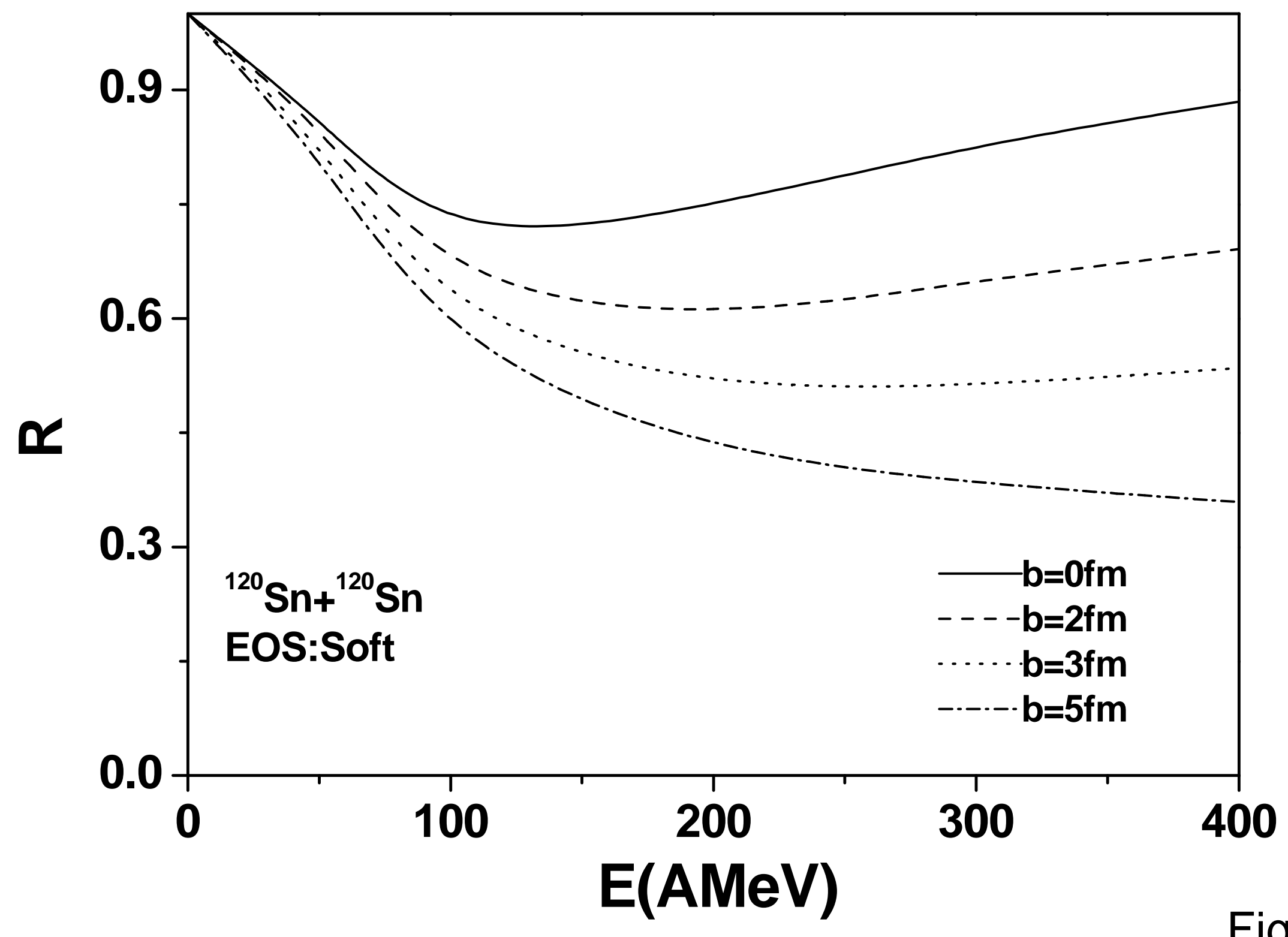

Fig. 4 